\title{
Rab6 is a Modulator of the Unfolded Protein Response: Implications for Alzheimer's Disease
}

\author{
Hyung Lim Elfrink ${ }^{\mathrm{a}}$, Rob Zwart ${ }^{\mathrm{a}}$, María L. Cavanillas ${ }^{\mathrm{a}}$, Adam Jay Schindler ${ }^{\mathrm{b}}$, Frank Baas ${ }^{\mathrm{a}, \mathrm{c}}$ \\ and Wiep Scheper ${ }^{\mathrm{a}, \mathrm{c}, *}$ \\ ${ }^{a}$ Department of Genome Analysis, Academic Medical Center, Amsterdam, The Netherlands \\ ${ }^{\mathrm{b}}$ Department of Biology, Duke University, Durham, North Carolina, NC, USA \\ ${ }^{\mathrm{c}}$ Department of Neurology, Academic Medical Center, Amsterdam, The Netherlands
}

Handling Associate Editor: Othman Ghribi

Accepted 1 November 2011

\begin{abstract}
The unfolded protein response (UPR) is a stress response of the endoplasmic reticulum (ER), the first compartment of the secretory pathway. The UPR is activated in non-tangle bearing neurons in Alzheimer's disease (AD) brain, indicating it is an early phenomenon. We found that the level of Rab6, implicated in anterograde and retrograde trafficking in the secretory pathway, is increased in brains of AD patients. Rab6 expression, closely correlated with the extent of UPR activation, is not controlled by the UPR. This suggests that Rab6 and UPR activation are both increased in response to early pathogenic changes in AD. Here we demonstrate that Rab6 modulates the UPR, increased levels inhibit whereas decreased levels augment UPR induction. Rab6 is not involved in the initial phase of the UPR; it only affects the UPR after prolonged ER stress. We propose that Rab6 is involved in the recovery from an ER stress insult. The increased Rab6 levels in AD brain in combination with UPR activation suggest that a failure to recover from ER stress may contribute to neurodegeneration in AD. The Rab6 mediated recovery pathway may provide a target to selectively inhibit the destructive pathways of the UPR.
\end{abstract}

Keywords: Alzheimer's disease, endoplasmic reticulum stress, Rab6, unfolded protein response

Supplementary data available online: http://dx.doi.org/10.3233/JAD-2011-110971

\section{INTRODUCTION}

For therapeutic intervention in Alzheimer's disease (AD), it is pivotal to target early pathogenic pathways. We reported activation of the unfolded protein response (UPR) as an early event in neurons in $\mathrm{AD}$ brain $[1,2]$. The UPR is a stress response of the endoplasmic reticulum (ER). Several homeo-

\footnotetext{
*Correspondence to: Wiep Scheper, Department of Genome Analysis, Academic Medical Center, P.O. Box 22660, 1100 DD Amsterdam, The Netherlands. Tel.: +31 20566 4959; Fax: +31 20 566 9312; E-mail: w.scheper@amc.uva.nl.
}

static and biosynthetic pathways are located in the ER: it is a site for $\mathrm{Ca}^{2+}$ homeostasis, redox balance, lipid synthesis, and, importantly, the synthesis and folding of membrane bound and secretory proteins. Disturbance of ER homeostasis leading to intraluminal protein misfolding (termed ER stress) results in activation of the UPR [3, 4]. The UPR signals via three sensor molecules in the ER membrane, doublestranded RNA-activated protein kinase (PKR)-like endoplasmic reticulum kinase (PERK), inositol requiring kinase 1 (Ire1), and activating transcription factor 6 (ATF6). These sensor molecules are bound by the 
ER chaperone binding immunoglobulin (heavy chain) protein/glucose-related protein 78 (BiP/GRP78) under normal conditions. In case of ER stress, BiP releases from the sensors and the three signaling pathways of the UPR are activated. The PERK pathway is involved in translational regulation. Activated PERK phosphorylates the translation initiation factor eIF $2 \alpha$ resulting in an overall attenuation of translation and selective translation of specific mRNAs by upregulation of activating transcription factor 4 (ATF4). Activation of Ire1 leads to splicing of the XBP1 mRNA, resulting in production of the active transcription factor XBP1 (spliced). Another transcription factor is produced by activation of ATF6: BiP release allows ATF6(-p90) to traffic to the Golgi, where it is sequentially processed by the site- 1 and site- 2 proteases to render the active ATF6-p50 transcription factor.

Activation of the UPR is initiated to restore homeostasis in the ER. The inhibition of protein synthesis is aimed to temporarily reduce the protein load in the ER. In addition, the expression of proteins that assist in protein folding (e.g., BiP) is increased [5, 6]. Also, the proteolysis of aberrant proteins is facilitated by upregulation of components of the ER associated degradation (ERAD) machinery and activation of autophagy [7-11]. Furthermore, the expression of pro-apoptotic proteins like cAMP response elementbinding $(\mathrm{C} / \mathrm{EBP})$ homologous protein $(\mathrm{CHOP})$ is increased. If the homeostasis is restored, the UPR will be switched off, but prolonged activation will result in cell death $[12,13]$.

During UPR activation the regular function of the ER as major protein factory and first compartment in the secretory pathway is compromised. This will affect several vesicle transport routes, which is particularly problematic for cells that are very dependent on transport of vesicles for proper function, like neurons. Rab proteins, members of the small GTPase super family, are important regulators of vesicle transport via interactions with effector proteins and motor proteins [14]. Rab6, in its active form a Golgi resident protein, has been implicated in retrograde and anterograde trafficking in the secretory pathway [15-19]. We have previously found that the levels of Rab6 are increased in AD temporal cortex, in close correlation with the extent of UPR activation [20]. We showed that Rab6 expression is not controlled by the UPR, suggesting that increased Rab6 and UPR are independent events in response to early pathogenic changes in AD. In this study we investigated the functional connection between Rab6 and the UPR in more depth. Our data suggest a novel pathway that may be employed to selectively modulate the destructive effects of prolonged ER stress, which is of potential interest for AD that is characterized by UPR activation early in the pathogenesis.

\section{MATERIALS AND METHODS}

\section{Materials}

Cell culture media and reagents were obtained from Gibco/Invitrogen (Carlsbad, CA, USA), plasmid selection reagents were from Invitrogen (Carlsbad, CA, USA) and other chemicals were from Sigma (St. Louis, MO, USA), unless indicated otherwise.

\section{Plasmids and generation of TREx-HeLa-Rab6 Q72R cell line}

Expression plasmids pSVSport1 Rab6A WT, -Q72R (constitutive active) and -T27N (dominant negative) [21] were a kind gift from C. C. Hoogenraad. All three Rab6A cDNAs were constructed to include a $5^{\prime}$ Myctag. The Myc-Rab6A Q72R cDNA was cloned into the pcDNA4/TO vector (Invitrogen) using EcoRI and XbaI restriction sites. TREx-HeLa cells (Invitrogen) were stably transfected with pcDNA4/TO Myc-Rab6A Q72R, according to manufacturer's protocol, to generate TREx-HeLa-Rab6 Q72R cell lines. Selection of double positive clones was performed with Blastidicin $(20 \mu \mathrm{g} / \mathrm{mL})$ and Zeocin $(1 \mathrm{mg} / \mathrm{mL})$. Single clones were selected and inducible expression of transgenic MycRab6A Q72R was verified on Western blot.

\section{Cell culture and treatment}

HeLa- and TREx-HeLa-Rab6 Q72R cells were cultured in Dulbecco's modified Eagle medium (DMEM) with GlutaMAX supplemented with $10 \%$ (v/v) fetal calf serum (FCS, Sigma), $100 \mathrm{U} / \mathrm{mL}$ penicillin and $100 \mu \mathrm{g} / \mathrm{mL}$ streptomycin. Cell culture medium for TREx-HeLa-Rab6 Q72R cells was additionally supplemented with $5 \mu \mathrm{g} / \mathrm{mL}$ Blasticidin and $400 \mu \mathrm{g} / \mathrm{mL}$ Zeocin to maintain plasmid selection. TREx-HeLaRab6 Q72R cells were plated in a 12 wells plate at a density of $2.10^{5}$ cells per well (on poly-L-lysine coated glass coverslips for immunofluorescence) and transgenic Myc-Rab6 Q72R expression was initiated by culturing in the presence of $1 \mu \mathrm{g} / \mathrm{mL}$ tetracycline (Tet) for at least $24 \mathrm{~h}$ before treatment. CHOATF6 cells were previously described [22] and were maintained in DMEM/Ham's F12 (1: 1) with GlutaMAX supplemented with $7.5 \%$ (v/v) FCS, $100 \mathrm{U} / \mathrm{mL}$ 
Table 1

Antibodies used for Western blot and immunofluorescence

\begin{tabular}{llll}
\hline Antibody & Species & Mono-/polyclonal & Manufacturer \\
\hline $\begin{array}{l}\text { For Western blotting } \\
\text { BiP/GRP78 }\end{array}$ & Goat & Polyclonal (N-20) & Cat. no. \\
c-Myc & Mouse & Monoclonal (9E10) & Boehringer Mannheim \\
eEF2 $\alpha$ & Rabbit & Polyclonal & Cell Signaling \\
Rab6 & Rabbit & Polyclonal (C-19) & Santa Cruz \\
FLAG & Mouse & Monoclonal (M2) & Sigma \\
Actin & Mouse & Monoclonal (AC-40) & Sigma \\
peIF2 $\alpha$ & Rabbit & Monoclonal (119A11) & Cell Signaling \\
For immunofluorescence & & & S1804 \\
CHOP/GADD153 & Mouse & Monoclonal (B-3) & Santa Cruz \\
CHOP/GADD153 & Mouse & Monoclonal (L63F7) & Cell Signaling \\
c-Myc-FITC & Mouse & Monoclonal (9E10) & Sigma \\
\hline
\end{tabular}

For Western blotting, incubations with peIF2 $\alpha$ were performed according to manufacturer's protocol, all other antibody incubations were performed $1: 1000$ in $2.5 \%(\mathrm{w} / \mathrm{v})$ milk in PBS-T $(0.05 \%, \mathrm{v} / \mathrm{v})$. Incubations for immunofluorescence with CHOP antibody from Cell Signaling was performed according to manufacturer's protocol, all other incubations were performed $1: 200 \mathrm{in} 1 \%$ (w/v) BSA, 0.05\% (w/v) saponin in PBS. The CHOP antibody from Cell Signaling was used for Figs. 1 and 2, and the antibody from Santa Cruz was used for supplementary Figure 1.

penicillin, $100 \mu \mathrm{g} / \mathrm{mL}$ streptomycin, and $250 \mu \mathrm{g} / \mathrm{mL}$ hygromycin-B to maintain stable $3 \times$ FLAG-ATF6 $^{2}$ expression. All cells were incubated at $37^{\circ} \mathrm{C}, 5 \%$ $\mathrm{CO}_{2}$ and $95 \%$ humidity. Cells were treated with tunicamycin at the indicated times and concentrations. To allow the cells to recover from tunicamycin induced ER stress, cells were washed once with complete medium without tunicamycin and subsequently incubated on complete medium without tunicamycin.

\section{Plasmid and siRNA transfection}

Transfections were performed with Lipofectamin 2000 Transfection Reagent (Invitrogen) according to manufacturer's protocol. For plasmid transfection, cells were seeded at a density of $8.10^{4}$ or $2.10^{5}$ cells per well (12 wells plate) for HeLa or CHO-ATF6 cells, respectively. Cells were transfected with $1.6 \mu \mathrm{g} / \mathrm{mL}$ plasmid DNA and treatment was started 24-36 h after transfection.

The siRNA duplexes were prepared by Sigma/ Proligo. The sequence of the scramble siRNA duplex was (sense: $5^{\prime}$ r(AGUACUGCUUACGAUA CGG)d(TT) $3^{\prime}$ ) and the sequences of Rab6 siRNA duplex 1 (sense: 5' r(GACAUCUUUGAUCACCA GA)d(TT) 3') and duplex 2 (sense: 5' r(CACCUA UCAGGCAACAAUU)d(TT) 3') were previously published elsewhere [23]. For Rab6 knockdown in HeLa cells duplex 1 was used and for Rab6 knockdown in CHO-ATF6 cells duplex 2 was used. For mRNA knockdown with siRNA, cells were seeded at a density of $2.10^{4}$ cells per well (12 wells plate). Cells were transfected with $25 \mathrm{nM}$ siRNA and treatment was started $72 \mathrm{~h}$ after knockdown initiation.

\section{SDS-PAGE and Western blotting}

Cells were harvested by scraping with a rubber policeman in 1\% (v/v) Triton X-100 PBS lysis buffer supplemented with protease inhibitors (either Leupeptin and PMSF, or Complete protease inhibitors from Roche, Penzberg, Germany). For the lysis buffer for peIF $2 \alpha$ Western blots, TBS replaced PBS and PhosSTOP (Roche) was added. Cell lysates were vigorously mixed, incubated on ice for $5 \mathrm{~min}$, and centrifuged for $5 \mathrm{~min}$ at $20.000 \times \mathrm{g}$ at $4^{\circ} \mathrm{C}$. Supernatant protein content was determined by Bio-Rad Protein Assay (Bio-Rad, Hercules, CA, USA). Equal amounts of protein were loaded in each lane on a gel. Cell lysates were separated on appropriate percentage polyacrylamide gels, $8 \%$ for $\mathrm{BiP}, 10 \%$ for ATF6 and peIF $2 \alpha$, and $12 \%$ for Rab6. Western blotting and analysis was performed as described previously [24]. The antibodies used in this study are listed in Table 1.

\section{Immunofluorescence}

For immunofluorescence stainings, cells were plated on glass coverslips. After treatment, cells were washed three times in ice-cold PBS, fixed in $4 \%(\mathrm{w} / \mathrm{v})$ paraformaldehyde, 4\% (w/v) sucrose (Merck, Darmstadt, Germany) in PBS for 5 min at room temperature, washed three times with ice-cold PBS, permeabilized 
with (pre-cooled) methanol for $5 \mathrm{~min}$ at $-20^{\circ} \mathrm{C}$, and washed three times with ice-cold PBS before immunostaining. The following steps were performed at room temperature. Coverslips with cells were blocked in $0.1 \%$ (w/v) BSA fraction $\mathrm{V}, 0.05 \%(\mathrm{w} / \mathrm{v})$ saponin in PBS for $30 \mathrm{~min}$. CHOP antibody (Table 1) incubation was performed in $1 \%(\mathrm{w} / \mathrm{v}) \mathrm{BSA}$ and $0.05 \%$ (w/v) saponin in PBS for $1 \mathrm{~h}$. Cells on glass slides were washed three times in block buffer before 30 min incubation with the Cy3 labeled secondary antibody (Jackson ImmunoResearch, West Grove, PA, USA) in the same buffer as the primary antibody. For detection of overexpressed Rab6, cells were washed and incubated (as described for primary antibodies) with directly labeled FITC anti-Myc after CHOP secondary antibody incubation. Cells on coverslips were washed three times in block buffer, before counterstain with 4',6-Diamidino-2-phenylindole dihydrochloride (DAPI, $1.3 \mu \mathrm{M}$ ) was applied to the cells for $5 \mathrm{~min}$. Coverslips were rinsed in PBS, air dried, and mounted on glass slides with Vectashield Mounting Medium (Vector Laboratories, Burlingame, CA, USA) and sealed with nail polish. Images were captured using a VANOX AHBT3 microscope (Olympus, Shinjuku, Tokyo, Japan) equipped with a digital camera. The CHOP positive nuclei from a seven fields of view per condition were quantified.

\section{RNA isolation and cDNA synthesis}

Cells were lysed and scraped in TRIzol Reagent (Invitrogen) and organic- and aqueous phase separation was performed by mixing with chloroform (Merck). Subsequent RNA isolation was performed either manually (according to the manufacturer's protocol) or automated with an RNeasy MiniKit on a Qiacube (Qiagen, Venlo, the Netherlands; according to manufacturer's protocol). RNA purity and integrity were assessed on a NanoDrop 2000 spectrophotometer (Thermo Scientific, Waltham, MA, USA) and on a $0.8 \%(\mathrm{w} / \mathrm{v})$ agarose gel $(2.5 \mu \mathrm{g} / \mathrm{mL}$ ethidium bromide).

cDNA synthesis was performed on $0.5-1.0 \mu \mathrm{g}$ of RNA per reaction (RNA sample quantities do not vary within experiments) using a SuperScript II Reverse Transcriptase Kit (Invitrogen). Priming of mRNA poly-A tails was performed with $125 \mathrm{pmol}$ oligo(dT) ${ }_{12}-\mathrm{VN}$ primer in a final volume of $10 \mu \mathrm{L}$ and was incubated at $70^{\circ} \mathrm{C}$ for $10 \mathrm{~min} . \mathrm{MgCl}_{2}(2 \mathrm{mM})$, dNTPs $(0.5 \mathrm{mM}$ each), $5 \mu \mathrm{L} 5 \times$ First-Strand Buffer and $100 \mathrm{U}$ SuperScript II reverse transcriptase were added to the primed mRNAs in a final volume of $25 \mu \mathrm{L}$ per reaction and incubated at $42^{\circ} \mathrm{C}$ for $1 \mathrm{~h}$. The reverse transcription reaction was stopped by incubation at $70^{\circ} \mathrm{C}$ for $10 \mathrm{~min}$.

\section{Real-time qPCR}

Per sample, $1 \mu \mathrm{L}$ cDNA was pipetted in triplicate into a 384 wells plate and dried in a DNA110 SpeedVac (Thermo Savant). qPCR mixtures were prepared as described previously [24]. Probe and primer combinations are listed in Table 2. qPCR was performed on a LightCycler 480 system (Roche) with the following PCR program: $10 \mathrm{~min}$ denaturation at $95^{\circ} \mathrm{C}$, followed by 45 cycles of denaturation at $95^{\circ} \mathrm{C}$ for $10 \mathrm{~s}$, annealing at $58^{\circ} \mathrm{C}$ for $20 \mathrm{~s}$ and elongation at $72^{\circ} \mathrm{C}$ for $1 \mathrm{~s}$ (including signal acquisition), the PCR program was finalized by a $10 \mathrm{~s}$ cool down at $40^{\circ} \mathrm{C}$. Results were analyzed using the LightCycler 480 software (version 1.5.0.39) and PCR efficiencies were calculated using LinRegPCR application (version 12.10, [25]). All data are presented as mean $\pm \mathrm{SD}$ from a single experiment. The magnitude of the normalized values between experiments may vary. For statistical analyses the unpaired two-tailed Student's $T$-test was performed. Differences were accepted as statistically significant at $p \leq 0.05$.

\section{RESULTS}

\section{Rab6 overexpression attenuates the UPR}

To investigate the effect of Rab6 function on the UPR, Rab6 was overexpressed in a HeLa cell model. Rab6 overexpression was analyzed by qPCR (Fig. 1A) and Western blot (Fig. 1B). Stimulation of the UPR was performed by treatment with increasing concentrations of tunicamycin for $20 \mathrm{~h}$. The UPR induced expression of $\mathrm{BiP}$ and $\mathrm{CHOP}$ is mediated by a transcriptional response. Therefore, the mRNA levels of the UPR markers $\mathrm{BiP}$ and $\mathrm{CHOP}$ were determined by qPCR. As expected, tunicamycin treatment upregulates $\mathrm{BiP}$ mRNA (Fig. 1C) and CHOP mRNA (Fig. 1D). Transient overexpression of Rab6 reduces BiP induction by $\sim 1.5$ fold in comparison to mock transfection, (Fig. 1C), which is most prominent at $0.2 \mu \mathrm{g} / \mathrm{mL}$ tunicamycin (mock $5.7 \pm 0.8$ versus Rab6 $3.7 \pm 0.3$ ). UPR induced CHOP mRNA levels are reduced 2- to 3-fold in cells overexpressing Rab6 (Fig. 1D), the strongest effect is observed at $0.5 \mu \mathrm{g} / \mathrm{mL}$ tunicamycin (mock $11.1 \pm 0.9$ versus Rab6 4.2 \pm 0.3 ).

To verify these findings on mRNA level, BiP and $\mathrm{CHOP}$ protein inductions were analyzed in the presence or absence of Rab6 overexpression. Tunicamycin treatment results in the induction of $\mathrm{BiP}$ as 
A

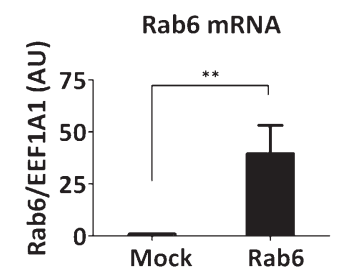

C

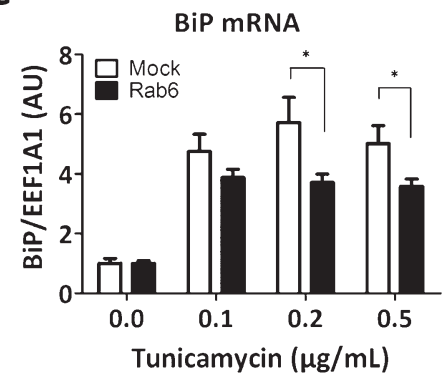

B<smiles>[R6]C1C#CC(=O)C1[R6]</smiles>

D

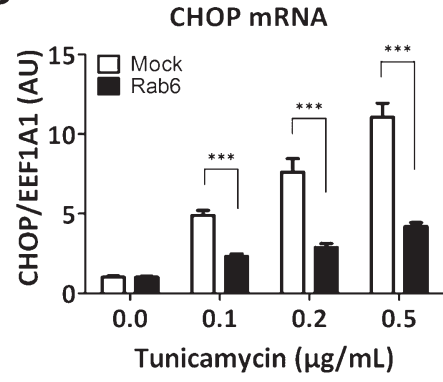

$\mathbf{E}$

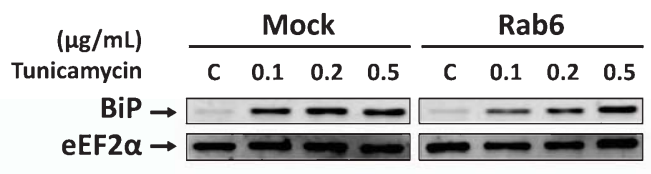

Fold change

$\begin{array}{llllllll}1 & 12 & 16 & 17 & 1 & 7 & 9 & 18\end{array}$

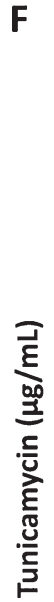

DAPI
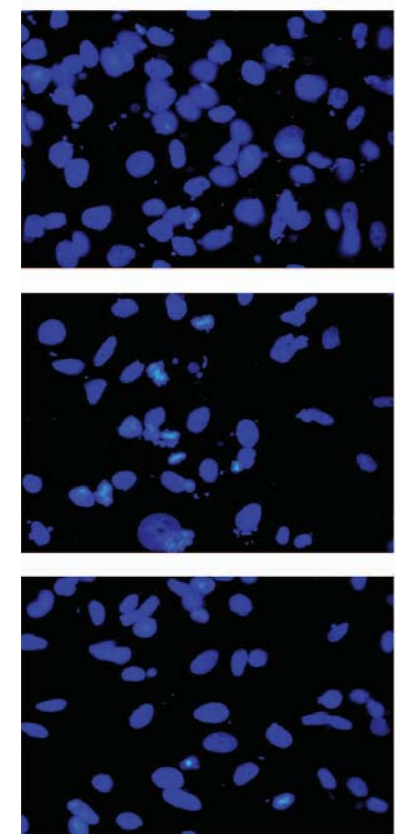

Myc-Rab6
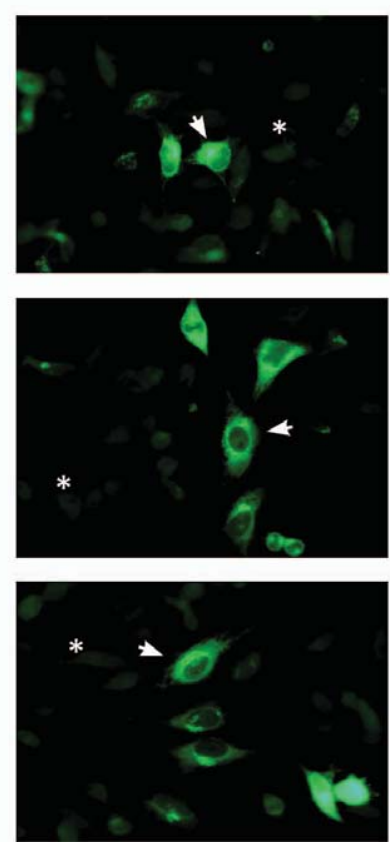

CHOP
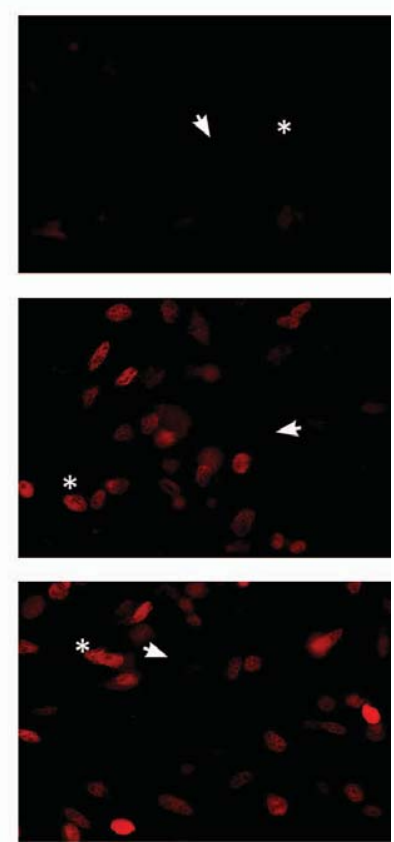

Fig. 1. Rab6 overexpression attenuates the UPR. HeLa cells were transfected with Rab6 or empty vector (mock). Rab6 overexpression was assessed by qPCR (A) and Western blotting (B). ER stress was induced by tunicamycin treatment for 20 hours as indicated. The effect of Rab6 overexpression on the relative mRNA expression of $\mathrm{BiP}(\mathrm{C})$ and $\mathrm{CHOP}(\mathrm{D})$ was assessed by qPCR. EEF1A1 was used as a reference gene. Shown are mean and SD in arbitrary units (AU; $n=3, * p \leq 0.05, * * p \leq 0.01$, and $* * * p \leq 0.001$ ). The effect of Rab6 overexpression on BiP protein expression (E) was analyzed by Western blotting. Equal amounts of protein were loaded in each lane and eEF2 $\alpha$ was used as a loading control. F) The effect of Rab6 overexpression on nuclear CHOP expression was assessed by immunofluorescence. Arrows indicate examples of cells that show high Rab6 overexpression and low CHOP levels, and asterisks indicate examples of cells that express low Rab6 levels and high CHOP levels. Nuclear counterstain was performed with DAPI. 
Table 2

Primer and probe combinations for $\mathrm{qPCR}$

\begin{tabular}{llc}
\hline Amplicon & Primers $\left(5^{\prime}-3^{\prime}\right)$ & Probe $^{1}$ \\
\hline Rab6 & Fw: GCACACAGGACAGAAGCAGA & $\# 75$ \\
& Rv: GGTTGAAGATGACATGGGAGAT & \\
BiP/GRP78 & Fw: CATCAAGTTCTTGCCGTTCA & \\
& Rv: TCTTCAGGAGCAAATGTCTTTGT & \\
CHOP/GADD153 & Fw: AAGGCACTGAGCGTATCATGT & \\
& Rv: TGAAGATACACTTCCTTCTTGAACA & \\
EEF1A1 & Fw: CAATGGCAAAATCTCACTGC & $\# 63$ \\
& Rv: AACCTCATCTCTATTAAAAACACCAAA \\
\hline
\end{tabular}

Primers were prepared by Sigma; ${ }^{1}$ Referring to Universal ProbeLibrary for Human probes from Roche.

determined by Western blotting (Fig. 1E). Rab6 overexpression clearly reduces the $\mathrm{BiP}$ protein induction in response to tunicamycin elicited ER stress (Fig. 1E). To determine the effect of Rab6 on CHOP protein expression, Rab6 overexpressing cells were identified by immunofluorescent staining, detecting the myctag of the exogenous Rab6 (Fig. 1F). Treatment with tunicamycin increases the number of $\mathrm{CHOP}$ positive nuclei, these are predominantly observed in cells which have low or no Rab6 transgene expression; CHOP expression is reduced in Rab6 overexpressing cells.

These results are supported by a similar experiment in TREx-HeLa Q72R, a stable cell line with inducible expression of Rab6 Q72R (a constitutive active mutant of Rab6). Rab6 Q72R overexpression induced by tetracylin (supplementary Figure 1A; available online: http://www.j-alz.com/issues/28/vol284.html\#supplementarydata07) is comparable to the overexpression in the transiently Rab6 (WT) transfected HeLa cells (Fig. 1B) and these cells are similarly responsive to tunicamycin treatment. Interestingly, constitutive active Rab6 also reduces BiP induction (supplementary Figure 1B). The number of CHOP positive nuclei after tunicamycin treatment was reduced from $28 \%$ to $19 \%$, by Rab6 Q72R overexpression, indicating that the nuclear translocation of CHOP is reduced (supplementary Figure 1C). These results further support the finding that Rab6 reduces UPR activation.

To investigate whether the effect of Rab6 overexpression on the UPR is protective or cytotoxic, HeLa cells were transfected with Rab6 and the tunicamycin induced toxicity was analyzed by phase contrast microscopy. This shows a concentration dependent cell loss as result of the tunicamycin treatment in the mock transfected cells (supplementary Figure 2). In contrast, the Rab6 overexpressing cells are more resistant to tunicamycin induced cell toxicity. This result indicates that increased Rab6 levels are protective during
ER stress, which correlates with the reduced expression of the pro-apoptotic protein CHOP in cells which overexpress Rab6.

\section{Rab6 knockdown increases the UPR}

Knockdown of Rab6 by siRNA transfection was performed in HeLa cells to test whether this could affect the UPR. Rab6 knockdown was analyzed by qPCR (Fig. 2A) and by Western blot (Fig. 2B). Induction of the UPR was established by $20 \mathrm{~h}$ treatment with different concentrations of tunicamycin. The response of the UPR was assessed on mRNA level by qPCR. Tunicamycin treatment increases the expression of the UPR markers BiP (Fig. 2C) and CHOP (Fig. 2D). Cells with reduced Rab6 levels, however, showed higher inductions of both BiP (Fig. 2C) and CHOP mRNA (Fig. 2D). BiP mRNA levels are increased 3-fold in Rab6 compared to scramble siRNA transfected cells at $0.2 \mu \mathrm{g} / \mathrm{mL}$ tunicamycin $(2.1 \pm 0.4$ versus $7.0 \pm 1.6$, mean \pm SD) and are 1.5-fold increased at $0.5 \mu \mathrm{g} / \mathrm{mL}$ tunicamycin ( $5.6 \pm 0.9$ versus $8.8 \pm 1.3$, Fig. $2 \mathrm{C})$. For CHOP mRNA a 2-fold augmentation is observed at $0.2 \mu \mathrm{g} / \mathrm{mL}$ tunicamycin $(8.3 \pm 1.3$ versus $18.3 \pm 4.5)$. The CHOP response levels out at $0.5 \mu \mathrm{g} / \mathrm{mL}$ tunicamycin (Fig. 2D).

The effect of Rab6 knockdown on the UPR was verified on protein level. BiP protein shows a dose dependent response to the tunicamycin treatment. As expected, Rab6 knockdown results in an additional increase of the $\mathrm{BiP}$ response (Fig. $2 \mathrm{E}$ ). $\mathrm{CHOP}$ nuclear localization was determined by immunofluorescense. Rab6 knockdown results in a stronger tunicamycin induced nuclear and overall CHOP staining as compared to scramble siRNA treated cells (Fig. 2F). These results show that Rab6 knockdown has an opposite effect on UPR activation compared to Rab6 overexpression, the UPR is increased upon Rab6 knockdown. Taken together, these data demonstrate that Rab6 
A

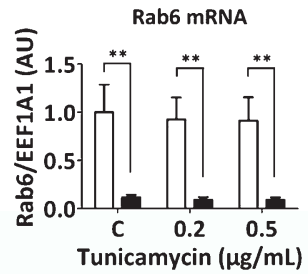

C

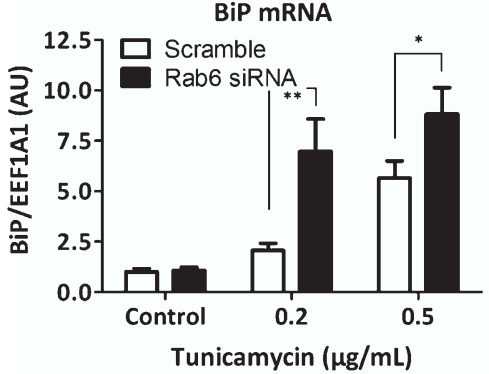

B

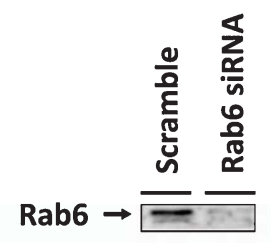

D

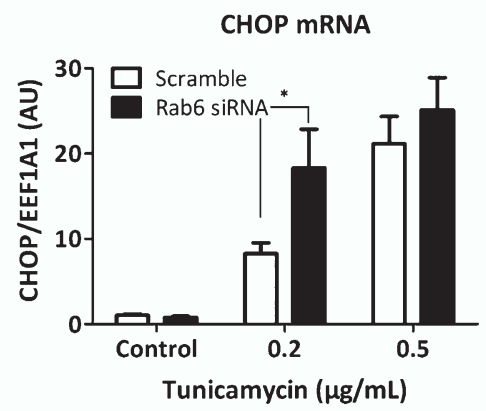

$\mathbf{E}$

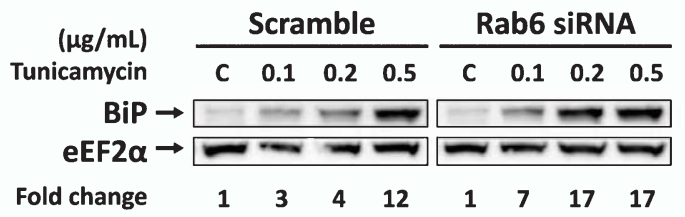

$\mathbf{F}$

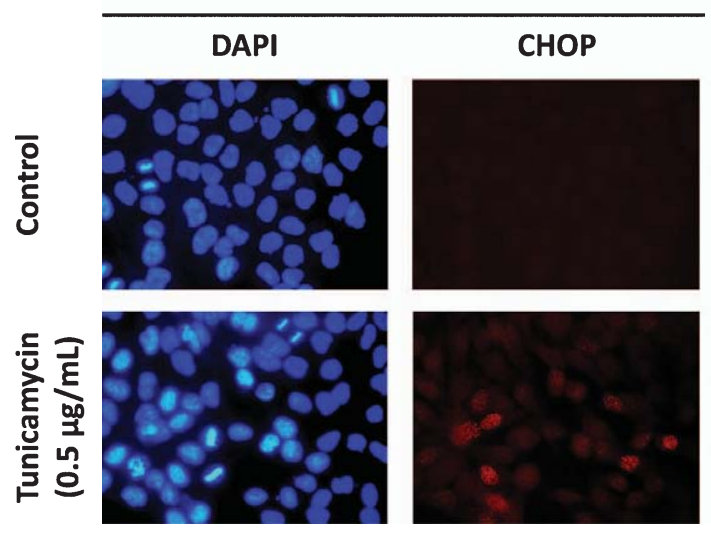

Rab6 siRNA

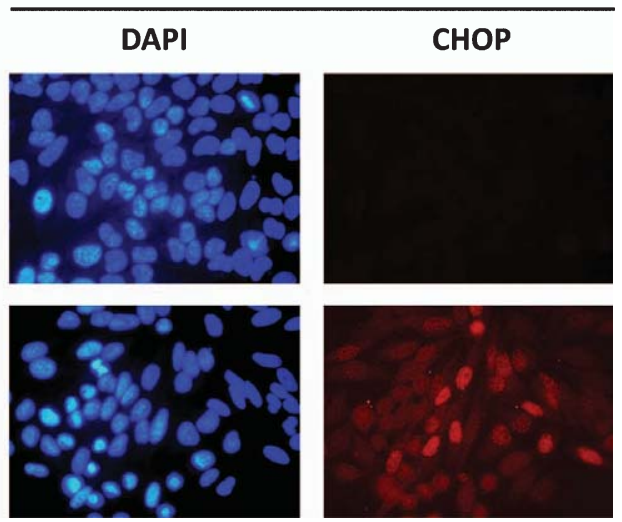

Fig. 2. Rab6 knockdown increases the UPR. HeLa cells were transfected with Rab6- and scramble siRNA. Rab6 knockdown was assessed by qPCR (A) and by Western blotting (B). ER stress was induced by treatment with tunicamycin for $20 \mathrm{~h}$ at the indicated concentrations. The effect of Rab6 knockdown on the relative mRNA expression levels for BiP (C) and CHOP (D) was determined. EEF1A1 was used as a reference gene. Shown are mean and SD in arbritary units (AU; $n=3,{ }^{*} p \leq 0.05$ and $* * p \leq 0.01$ ). E) The effect of knockdown of Rab6 on the BiP protein expression was assessed on Western blot. Equal amounts of protein were loaded in each lane and eEF2 $\alpha$ was used as a loading control. F) The effect of Rab6 knockdown on the UPR induced CHOP protein expression was assessed by immunofluorescence. Nuclei were counterstained with DAPI.

function has a negative modulatory effect on the UPR.

\section{Rab6 has no effect on the initial phase of the UPR}

Rab6 may exert its modulating effect directly on the UPR signaling pathways or on a feedback mechanism on the UPR. Out of the three branches of the UPR, the ATF6 pathway is the most likely to be influenced by Rab6 action. The generation of the active ATF6 transcription factor (ATF6-p50) is preceded by proteolytic processing of full-length ATF6(-p90) for which transport to the Golgi apparatus is necessary. To investigate whether Rab6 affects ATF6 processing, 

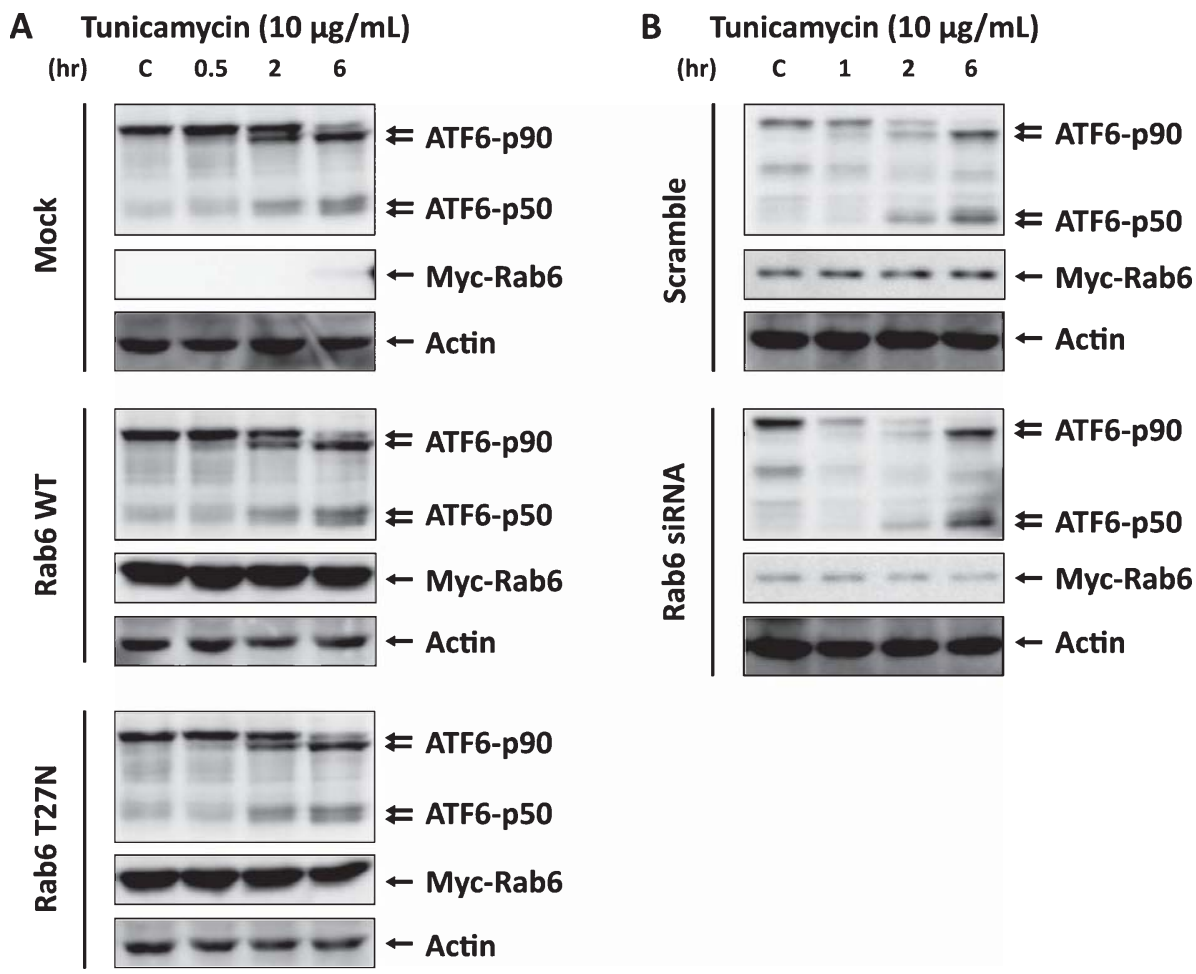

Fig. 3. Rab6 has no effect on ATF6 processing. CHO-ATF6 cells were transfected with Rab6 WT, Rab6 T27N (dominant negative) and empty vector (Mock; A), or were transfected with Rab6- and scramble siRNA (B). Cells were stimulated with $10 \mu \mathrm{g} / \mathrm{mL}$ tunicamycin for the indicated times, up to $6 \mathrm{~h}$. A, B) The induction of the ATF6 transcription factor (ATF6-p50) is assessed by Western blot analysis. The full-length ATF6 (ATF6-p90) shows as two bands, the upper represents C-terminal glycosylated ATF6 and the lower unglycosylated ATF6. The ATF6-p50 also shows as a doublet, the upper band probably represents the partially processed ATF6-p50 and the lower represents fully processed ATF6-p50. Equal amounts of protein were loaded in each lane and actin was used as a loading control.

a CHO cell model was used that stably expresses an N-terminal 3xFLAG tagged ATF6. High concentrations of tunicamycin are usually very toxic for prolonged incubations, however, shorter incubations allow the use of higher tunicamycin concentrations. The production of ATF6-p50 is a rapid event that is maximal after $8 \mathrm{~h}$ of stimulation with tunicamycin, and ATF6-p50 is no longer observed after prolonged stimulation [22]. In addition, this treatment protocol speeds up ATF6 processing and thereby facilitates the study of this initial signaling event of the UPR under Rab6 overexpression (supplementary Figure 3).

Rab6 WT and -T27N (dominant negative mutant of Rab6) were overexpressed and the induction of ATF6p50 by treatment with tunicamycin for up to $6 \mathrm{~h}$ was monitored by Western blot analysis (Fig. 3A). Comparison of the levels and kinetics of ATF6-p50 between mock, Rab6 WT and -T27N transfected cells shows no differences (Fig. 3A). Consistently, knockdown of Rab6 also has no effect on the induction of ATF6-p50 (Fig. 3B).
Another branch of the UPR is the PERK pathway. The downstream effector of PERK is eIF $2 \alpha$, which is phosphorylated to relieve the ER of its translational burden. The induction of phosphorylated $\operatorname{eIF} 2 \alpha$ (peIF $2 \alpha$ ) by tunicamycin is therefore a measure for activity of the PERK pathway. Rab6 overexpression and knockdown was performed in HeLa cells. After treatment with tunicamycin for $6 \mathrm{~h}$ with a broad range of tunicamyin concentrations, peIF $2 \alpha$ induction was analyzed on Western blot. The results show that a dose-dependent response of eIF $2 \alpha$ phosphorylation is observed, but there are no differences between mock and Rab6 transfected cells (Fig. 4A) nor between scramble and Rab6 siRNA transfected cells (Fig. 4B).

These results demonstrate that Rab6 has no effect on the processing of ATF6 and no effect on the phosphorylation of eIF $2 \alpha$. This indicates that the effect of Rab6 on the UPR does not target the initial signaling of the UPR. This is further supported by the finding that both Rab6 overexpression and knockdown does 

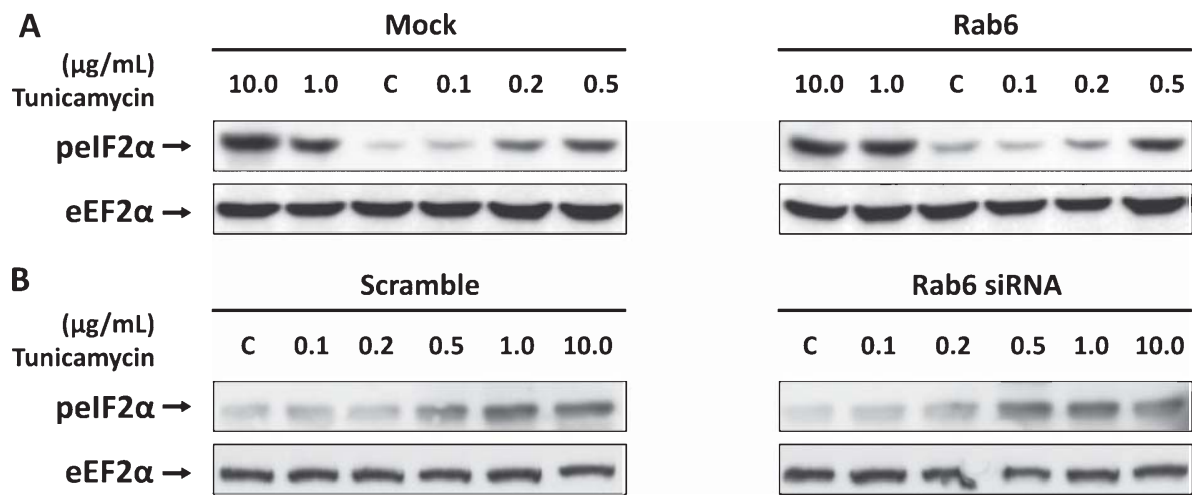

Fig. 4. Rab6 has no effect on the activation of the PERK pathway. HeLa cells were transfected with Rab6 and empty vector (A), or with Rab6 and scramble siRNA (B). The cells were treated with tunicamycin as indicated and peIF2 $\alpha$ induction was assessed on Western blot. Equal amounts of protein were loaded in each lane and eEF2 $\alpha$ was used as a loading control.

A

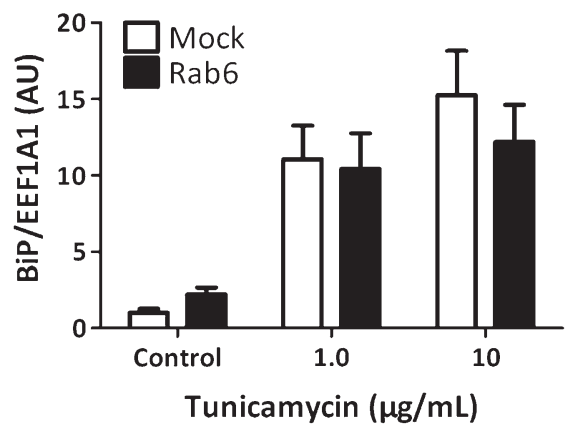

C

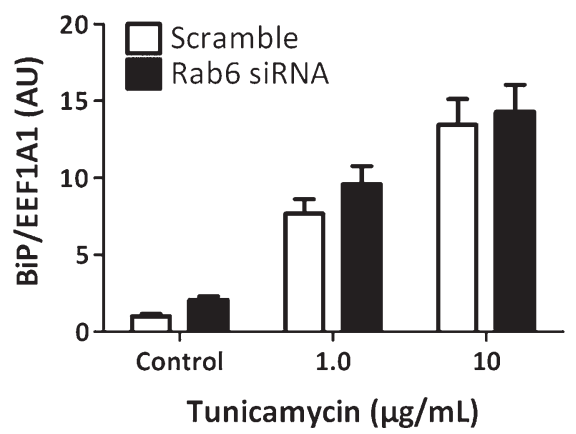

B

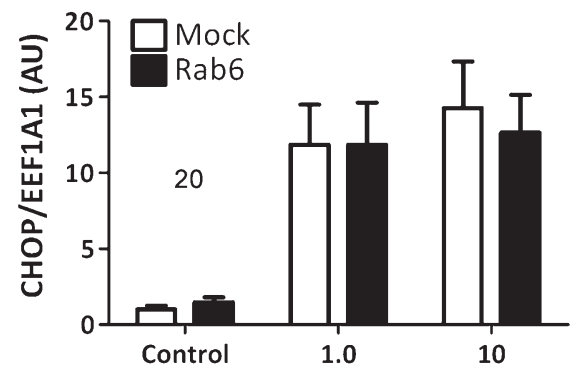

Tunicamycin $(\mu \mathrm{g} / \mathrm{mL})$

D

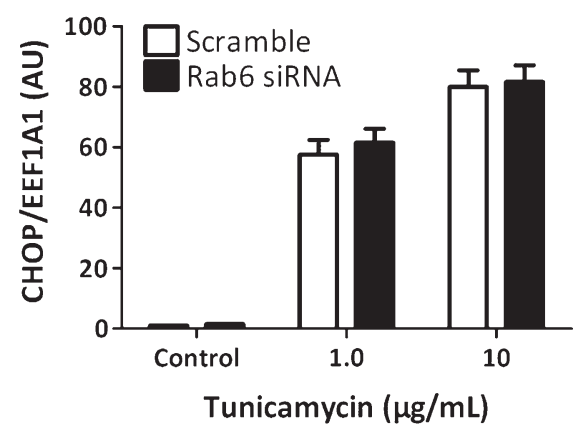

Fig. 5. Rab6 has no effect on the initial phase of the UPR. HeLa cells were transfected with Rab6 and empty vector (mock; A, B), or with Rab6and scramble siRNA (C, D). Cells were treated with 1.0 and $10.0 \mu \mathrm{g} / \mathrm{mL}$ tunicamycin for $6 \mathrm{~h}$ to induce ER stress. Relative expression of BiP $(\mathrm{A}, \mathrm{C})$ and CHOP mRNA (B, D) was determined by qPCR. EEF1A1 was used as a reference gene. Shown are mean and SD in arbitrary units (AU; $n=3$, there are no statistically significant differences).

not affect BiP and CHOP mRNA levels within $6 \mathrm{~h}$ of treatment with tunicamycin (Fig. 5). These results strongly suggest that Rab6 has no effect in the early phase of the UPR and is likely to function downstream of initiation.

\section{Rab6 knockdown impairs recovery of the UPR}

To test whether Rab6 affects the UPR via a feedback mechanism, a short tunicamycin pulse treatment was provided and recovery of the UPR was analyzed. 

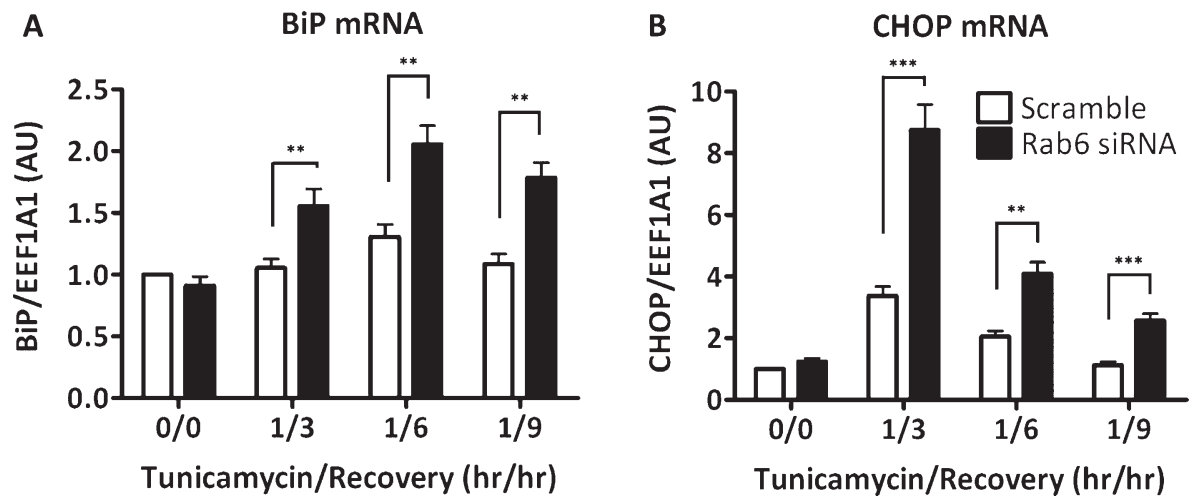

Fig. 6. Rab6 knockdown impairs recovery of the UPR. HeLa cells were transfected with Rab6- and scramble siRNA. Cells were stimulated with $1 \mu \mathrm{g} / \mathrm{mL}$ tunicamycin for $1 \mathrm{~h}$ and cells were washed with complete medium without tunicamycin and allowed to recover from ER stress for 3,6 , and $9 \mathrm{~h}$ on complete culture medium without tunicamycin, indicated by $1 / 3,1 / 6$, and $1 / 9$, respectively ( $0 / 0$ indicates the control). Relative expression of $\mathrm{BiP}(\mathrm{A})$ and $\mathrm{CHOP}$ mRNA (B) were analyzed by qPCR. EEF1A1 was used as a reference gene. Shown are mean and SD in arbitrary units (AU; $n=3, * * p \leq 0.01$ and $* * * p \leq 0.001)$.

HeLa cells transfected with scramble or Rab6 siRNA were treated for $1 \mathrm{~h}$ with tunicamycin $(1 \mu \mathrm{g} / \mathrm{mL})$ and allowed to recover from the stressor for 3,6 , and $9 \mathrm{~h}$, designated $1 / 3,1 / 6$, and $1 / 9$, respectively. The induction of BiP and CHOP mRNA was analyzed by qPCR. BiP induction is at control level after $3 \mathrm{~h}$ recovery in the scramble siRNA situation (Fig. 6A). In contrast, the BiP response for Rab6 knockdown cells remains increased $\sim 1.5$ fold compared to scramble siRNA transfected cells, irrespective of recovery time (Fig. 6A).

In the scramble siRNA transfected cells, CHOP is still 3-fold (3.4 \pm 0.3$)$ higher than control after $3 \mathrm{~h}$ recovery. Nine hours of recovery is sufficient for $\mathrm{CHOP}$ mRNA to return to normal levels (Fig. 6B). In the Rab6 knockdown cells, the CHOP mRNA level decreases during recovery, however, it remains 2- to 2.5 fold higher than in the scramble siRNA cells and is still not completely recovered after $9 \mathrm{~h}$ (Fig. 6B).

These results show that during recovery from an ER stress insult, BiP and CHOP mRNA levels are increased in Rab6 knockdown cells. These results indicate that Rab6 knockdown impairs recovery from ER stress and suggests a functional role for Rab6 in a feedback mechanism on the UPR.

\section{DISCUSSION}

In this study we investigated the functional connection between Rab6 and the UPR. We find that increased Rab6 levels decrease the output of the UPR, and conversely that decreased Rab6 levels augment
UPR induction, as shown on mRNA and protein level. We have also shown that this effect of Rab6 does not interfere early in the signaling response of the ATF6 (which requires vesicle transport) and PERK pathways. Although we cannot formally exclude that Rab6 affects the Ire1 signaling pathway, our combined data strongly indicate that Rab6 mediates its effect on the UPR after the initiation. This is corroborated by experiments where the recovery from an ER stress insult was shown to be impaired in the absence of Rab6.

How the UPR shuts down after restoration of homeostasis is not completely elucidated. One mechanism comprises the induction of $\mathrm{BiP}$ levels by the UPR, which limits the activation of the three ER stress sensors [26]. Furthermore, the ER stress responsive GADD43 facilitates the dephosphorylation of eIF2 $\alpha$ and thus provides a negative feedback in the PERK pathway [27, 28]. Recently, the kinase activity of yeast Ire1 was shown to be critical to for attenuation of its own activity [29, 30]. In addition to these UPR mediated feedback mechanisms, our data indicate that regulation of vesicle transport is an additional way to attenuate the UPR.

Rab6 has been shown to be involved in several antero- and retrograde trafficking pathways [15, 17-19]. Interestingly, Rab6 was shown to assist in the fission of vesicles from the Golgi, which may explain to some extent the broad range of Rab6 mediated trafficking pathways [31]. Although we cannot exclude that Rab6 has other yet unknown functions as well, it is likely that the inhibitory effect of Rab6 on the UPR is mediated directly by its function in vesicle transport. The retrograde Golgi-ER Rab6 pathway has been 
reported to transport bacterial toxins that are then subjected to translocation out of the ER [17]. This would be a way to target material from non-ER compartments for ERAD, but how this would relieve ER stress is not clear. Furthermore, retrograde membrane delivery via this Rab6 pathway may contribute to ER expansion, and this is shown to alleviate ER stress [32]. Transport out of the ER will reduce the protein load and may be a manner to relieve the stress. Rab1 was shown to function in this way to resolve ER stress in models for Parkinson's disease [33]. Alternatively, Rab6 mediated transport may facilitate an autophagic process. This is an interesting option in view of our recent data that during UPR activation, autophagy is the major degradational pathway [24]. Rab1 was recently implicated in the formation of autophagosomes from the ER in response to $\alpha$-synuclein [34], indicating the involvement of Rab proteins in regulated autophagy. In addition, an autophagy-like process was shown to be involved in the regulation of ERAD [35]. The precise trafficking pathway that is involved in the modulation of the UPR will be the subject of future investigation.

The UPR is an important protective stress mechanism that may become destructive if not adequately regulated. It appears that if ER homeostasis is restored, the apoptotic pathway is the most important process to limit. Our data indicate that the expression of the major UPR regulated pro-apoptotic factor, $\mathrm{CHOP}$, is strongly affected by Rab6. This suggests that the negative effects of prolonged ER stress can be targeted via Rab6. Increasing the function of Rab6 therefore allows modulation of the UPR without directly interfering in the UPR signaling. However, the UPR is activated despite high Rab6 levels in AD neurons. The increased Rab6 expression is predominantly found in non-tangle bearing neurons which have diffuse phosphorylated tau in AD brain, opposed by tangle bearing neurons which lack this increased Rab6 expression [20]. We therefore suggest that Rab6 levels are indeed increased as a protective response; this is supported by the data that show that Rab6 protects against ER stress mediated toxicity. Even though our current data provide evidence from non-neuronal cell based experiments, the UPR is a universal homeostatic response which is found in all cells, including neurons.

Since Rab6 is not induced by the UPR [20], it remains elusive what the trigger is to increase Rab6 levels. It is possible that the pathological state in $\mathrm{AD}$ forces the increased levels and that under more physiological UPR conditions Rab6 is regulated at a different level. For example, a rapid regulatory mechanism is provided by the GDP-GTP switch that controls the activity of Rab proteins; active GTP-bound Rab6 is associated with membranes, whereas GDP-bound Rab6 is inactive and localized in the cytosol $[14,36]$.

The question still remains why the UPR activation is not resolved by the high Rab6 levels in AD neurons. The answer may be downstream of the trafficking such as the impairment of the autophagy/lysosomal system as observed early in AD pathogenesis [37]. In AD hippocampus, we observe the activated UPR predominantly in neurons that have disturbances in the autophagy/lysosomal system [2, 24]. Recently a role for presenilin in lysosomal function was reported [38], which may provide an interesting connection to our previous work that indicated that the membrane association of Rab6 is dependent on presenilin 1 [39]. Further studies are required to establish how Rab6 attenuates the UPR and which part of the Rab6 mediated recovery pathway is dysfunctional in AD. This may lead to a novel target for intervention in neurodegenerative diseases that are associated with UPR activation, like AD.

\section{ACKNOWLEDGMENTS}

This study was supported by grants from the Internationale Stichting Alzheimer Onderzoek Nederland (ISAO \#07506) and the Netherlands Organisation for Scientific Research (NWO Life Sciences \# 836.05.060) to WS. We thank Line De Kimpe and Diana Nijholt for stimulating discussions.

Authors' disclosures available online (http://www.jalz.com/disclosures/view.php?id=1051).

\section{REFERENCES}

[1] Hoozemans JJ, Veerhuis R, van Haastert ES, Rozemuller JM, Baas F, Eikelenboom P, Scheper W (2005) The unfolded protein response is activated in Alzheimer's disease. Acta Neuropathol 110, 165-172.

[2] Hoozemans JJ, van Haastert ES, Nijholt DA, Rozemuller AJ, Eikelenboom P, Scheper W (2009) The unfolded protein response is activated in pretangle neurons in Alzheimer's disease hippocampus. Am J Pathol 174, 1241-1251.

[3] Kaufman RJ (1999) Stress signaling from the lumen of the endoplasmic reticulum: Coordination of gene transcriptional and translational controls. Genes Dev 13, 1211-1233.

[4] Ron D, Walter P (2007) Signal integration in the endoplasmic reticulum unfolded protein response. Nat Rev Mol Cell Biol 8, 519-529.

[5] Yoshida H, Haze K, Yanagi H, Yura T, Mori K (1998) Identification of the cis-acting endoplasmic reticulum stress response element responsible for transcriptional induction of mammalian glucose-regulated proteins. Involvement of basic 
leucine zipper transcription factors. J Biol Chem 273, 3374133749 .

[6] Yamamoto K, Yoshida H, Kokame K, Kaufman RJ, Mori K (2004) Differential contributions of ATF6 and XBP1 to the activation of endoplasmic reticulum stress-responsive cisacting elements ERSE, UPRE and ERSE-II. J Biochem 136, 343-350.

[7] Travers KJ, Patil CK, Wodicka L, Lockhart DJ, Weissman JS, Walter P (2000) Functional and genomic analyses reveal an essential coordination between the unfolded protein response and ER-associated degradation. Cell 101, 249258.

[8] Ng DT, Spear ED, Walter P (2000) The unfolded protein response regulates multiple aspects of secretory and membrane protein biogenesis and endoplasmic reticulum quality control. J Cell Biol 150, 77-88.

[9] Yorimitsu T, Nair U, Yang Z, Klionsky DJ (2006) Endoplasmic reticulum stress triggers autophagy. J Biol Chem 281, 30299-30304.

[10] Ogata M, Hino S, Saito A, Morikawa K, Kondo S, Kanemoto S, Murakami T, Taniguchi M, Tanii I, Yoshinaga K, Shiosaka S, Hammarback JA, Urano F, Imaizumi K (2006) Autophagy is activated for cell survival after endoplasmic reticulum stress. Mol Cell Biol 26, 9220-9231.

[11] Ding WX, Ni HM, Gao W, Hou YF, Melan MA, Chen X, Stolz DB, Shao ZM, Yin XM (2007) Differential effects of endoplasmic reticulum stress-induced autophagy on cell survival. J Biol Chem 282, 4702-4710.

[12] Wang XZ, Lawson B, Brewer JW, Zinszner H, Sanjay A, Mi LJ, Boorstein R, Kreibich G, Hendershot LM, Ron D (1996) Signals from the stressed endoplasmic reticulum induce C/EBP-homologous protein (CHOP/GADD153). Mol Cell Biol 16, 4273-4280.

[13] Zinszner H, Kuroda M, Wang X, Batchvarova N, Lightfoot RT, Remotti H, Stevens JL, Ron D (1998) CHOP is implicated in programmed cell death in response to impaired function of the endoplasmic reticulum. Genes Dev 12, 982-995.

[14] Hutagalung AH, Novick PJ (2011) Role of Rab GTPases in membrane traffic and cell physiology. Physiol Rev 91, 119149 .

[15] Martinez O, Antony C, Pehau-Arnaudet G, Berger EG, Salamero J, Goud B (1997) GTP-bound forms of rab6 induce the redistribution of Golgi proteins into the endoplasmic reticulum. Proc Natl Acad Sci U S A 94, 1828-1833.

[16] Girod A, Storrie B, Simpson JC, Johannes L, Goud B, Roberts LM, Lord JM, Nilsson T, Pepperkok R (1999) Evidence for a COP-I-independent transport route from the Golgi complex to the endoplasmic reticulum. Nat Cell Biol 1, 423430.

[17] White J, Johannes L, Mallard F, Girod A, Grill S, Reinsch S, Keller P, Tzschaschel B, Echard A, Goud B, Stelzer EH (1999) Rab6 coordinates a novel Golgi to ER retrograde transport pathway in live cells. J Cell Biol 147, 743-760.

[18] Darchen F, Goud B (2000) Multiple aspects of Rab protein action in the secretory pathway: Focus on Rab3 and Rab6. Biochimie 82, 375-384.

[19] Grigoriev I, Splinter D, Keijzer N, Wulf PS, Demmers J, Ohtsuka T, Modesti M, Maly IV, Grosveld F, Hoogenraad CC, Akhmanova A (2007) Rab6 regulates transport and targeting of exocytotic carriers. Dev Cell 13, 305-314.

[20] Scheper W, Hoozemans JJ, Hoogenraad CC, Rozemuller AJ, Eikelenboom P, Baas F (2007) Rab6 is increased in Alzheimer's disease brain and correlates with endoplasmic reticulum stress. Neuropathol Appl Neurobiol 33, 523532.
[21] Matanis T, Akhmanova A, Wulf P, Del NE, Weide T, Stepanova T, Galjart N, Grosveld F, Goud B, De Zeeuw CI, Barnekow A, Hoogenraad CC (2002) Bicaudal-D regulates COPI-independent Golgi-ER transport by recruiting the dynein-dynactin motor complex. Nat Cell Biol 4, 986992.

[22] Schindler AJ, Schekman R (2009) In vitro reconstitution of ER-stress induced ATF6 transport in COPII vesicles. Proc Natl Acad Sci U S A 106, 17775-17780.

[23] Young J, Stauber T, Del NE, Vernos I, Pepperkok R, Nilsson T (2005) Regulation of microtubule-dependent recycling at the trans-Golgi network by Rab6A and Rab6A'. Mol Biol Cell 16 162-177.

[24] Nijholt DA, de Graaf TR, van Haastert ES, Oliveira AO, Berkers CR, Zwart R, Ovaa H, Baas F, Hoozemans JJ, Scheper W (2011) Endoplasmic reticulum stress activates autophagy but not the proteasome in neuronal cells: Implications for Alzheimer's disease. Cell Death Differ 18, 10711081.

[25] Ruijter JM, Ramakers C, Hoogaars WM, Karlen Y, Bakker O, van den Hoff MJ, Moorman AF (2009) Amplification efficiency: Linking baseline and bias in the analysis of quantitative PCR data. Nucleic Acids Res 37, e45.

[26] Zhang K, Kaufman RJ (2004) Signaling the unfolded protein response from the endoplasmic reticulum. J Biol Chem 279, 25935-25938.

[27] Novoa I, Zeng H, Harding HP, Ron D (2001) Feedback inhibition of the unfolded protein response by GADD34-mediated dephosphorylation of eIF2alpha. J Cell Biol 153, 10111022.

[28] Ma Y, Hendershot LM (2003) Delineation of a negative feedback regulatory loop that controls protein translation during endoplasmic reticulum stress. J Biol Chem 278, 34864-34873.

[29] Rubio C, Pincus D, Korennykh A, Schuck S, El-Samad H, Walter P (2011) Homeostatic adaptation to endoplasmic reticulum stress depends on Ire1 kinase activity. J Cell Biol 193, 171-184.

[30] Chawla A, Chakrabarti S, Ghosh G, Niwa M (2011) Attenuation of yeast UPR is essential for survival and is mediated by IRE1 kinase. J Cell Biol 193, 41-50.

[31] Miserey-Lenkei S, Chalancon G, Bardin S, Formstecher E, Goud B, Echard A (2010) Rab and actomyosin-dependent fission of transport vesicles at the Golgi complex. Nat Cell Biol 12, 645-654.

[32] Schuck S, Prinz WA, Thorn KS, Voss C, Walter P (2009) Membrane expansion alleviates endoplasmic reticulum stress independently of the unfolded protein response. J Cell Biol 187, 525-536.

[33] Cooper AA, Gitler AD, Cashikar A, Haynes CM, Hill KJ, Bhullar B, Liu K, Xu K, Strathearn KE, Liu F, Cao S, Caldwell KA, Caldwell GA, Marsischky G, Kolodner RD, Labaer J, Rochet JC, Bonini NM, Lindquist S (2006) Alpha-synuclein blocks ER-Golgi traffic and Rab1 rescues neuron loss in Parkinson's models. Science 313, 324-328.

[34] Winslow AR, Chen CW, Corrochano S, Acevedo-Arozena A, Gordon DE, Peden AA, Lichtenberg M, Menzies FM, Ravikumar B, Imarisio S, Brown S, O'Kane CJ, Rubinsztein DC (2010) alpha-Synuclein impairs macroautophagy: Implications for Parkinson's disease. J Cell Biol 190, 10231037.

[35] de Haan CA, Molinari M, Reggiori F (2010) Autophagyindependent LC3 function in vesicular traffic. Autophagy 6 , 994-996.

[36] Martinez O, Goud B (1998) Rab proteins. Biochim Biophys Acta 1404, 101-112. 
[37] Boland B, Kumar A, Lee S, Platt FM, Wegiel J, Yu WH, Nixon RA (2008) Autophagy induction and autophagosome clearance in neurons: Relationship to autophagic pathology in Alzheimer's disease. J Neurosci 28, 6926-6937.

[38] Lee JH, Yu WH, Kumar A, Lee S, Mohan PS, Peterhoff CM, Wolfe DM, Martinez-Vicente M, Massey AC, Sovak G, Uchiyama Y, Westaway D, Cuervo AM, Nixon RA (2010)
Lysosomal proteolysis and autophagy require presenilin 1 and are disrupted by Alzheimer-related PS1 mutations. Cell 141, 1146-1158.

[39] Scheper W, Zwart R, Baas F (2004) Rab6 membrane association is dependent of Presenilin 1 and cellular phosphorylation events. Brain Res Mol Brain Res 122, 17-23. 